Pacific Journal of Mathematics

INVARIANT FUNCTIONS OF AN ITERATIVE PROCESS FOR 


\title{
INVARIANT FUNCTIONS OF AN ITERATIVE PROCESS FOR MAXIMIZATION OF A POLYNOMIAL
}

\author{
Peter F. Stebe
}

Let $P$ be a polynomial with real non-negative coefficients and variables $x_{i, j}, i=1, \cdots, k, j=1, \cdots, n_{i}$. Let $d=\sum_{1}^{k} n_{i}$. Let $R_{d}$ be the $d$-dimensional real vector space. Let $\tilde{M}$ be the subset of $R_{d}$ defined by

$$
\tilde{M}=\left\{x \mid x \in R_{d}, x_{i, j} \geqq 0, \sum_{j=1}^{n_{i}} x_{i, j}=1\right\}
$$

where the symbols $x_{i, j}$ denote the components of $x$. If $x$ is a vector in the interior of $\tilde{M}$, define $\tau(x)$ as the vector in $\tilde{M}$ with components $x_{i, j}^{\prime}$ given by

$$
x_{i, j}^{\prime}=\frac{x_{i, j} \frac{\partial P}{\partial x_{i, j}}}{\sum_{h=1}^{n_{i}} x_{i, h} \frac{\partial P}{\partial x_{i, h}}} .
$$

The expression on the right is evaluated at $x$. The transformation $\tau$ is defined on the boundary of $\tilde{M}$ by the same formula if the denominators do not vanish.

Let $\widetilde{F}$ be the set of fixed points of $\tau$ in $\tilde{M}$. It is shown that if $\tau$ is a homeomorphism of $\tilde{M}$ onto itself, there is a set of $d-k$ functions $f_{1}, \cdots, f_{d-k}$ defined on $\tilde{M}-\tilde{F}$ such that $f_{i}(x)=f_{i}(\tau(x))$ for $x \in \widetilde{M}-\widetilde{F}$. The functions $f_{i}$ are continuous and independent on an open dense subset of $\tilde{M}-\tilde{F}$. Explicit expressions for certain invariant functions are also obtained.

1. The transformation $\tau$. The transformation $\tau$ defined in the introduction can be used to iteratively find local maxima for the polynomial $P$. It was shown by L. E. Baum and J. A. Eagon [1] that if $P$ is a homogeneous polynomial with positive coefficients and if $x$ is an element of $\tilde{M}$ such that $\tau(x)$ is defined then either $\tau(x)=(x)$ or $P(\tau(x))>P(x)$. This result was generalized at the suggestion of 0 . Rothaus by L. E. Baum and G. R. Sell [2] to arbitrary polynomials with positive coefficients.

It will be assumed in this paper that the transformation $\tau$ is a homeomorphism of $\widetilde{M}$ onto itself. According to an unpublished result of L. E. Baum, $\tau$ is a homeomorphism of $\widetilde{M}$ onto itself if and only if the expression for $P$ as a sum of distinct monomials with positive coefficients contains monomials $c_{i, j} x_{i, j}{ }^{w_{i}, j}$ for all $i=1, \cdots, k, j=1, \cdots, n_{i}$ where $c_{i, j}>0$ and $w_{i, j}$ is an integer greater than zero. Since this condition is satisfied if and only if $\tau$ is defined on all of $\tilde{M}$, a necessary and sufficient condition that $\tau$ is a homeomorphism of $\tilde{M}$ onto itself 
is that $\tau$ be defined an all of $\tilde{M}$. We will not prove L. E. Baum's result here, but will give a single example of a polynomial $P$ for which $\tau$ is a homeomorphism. Let

$$
P=\sum_{i=1}^{k} \sum_{j=1}^{n_{i}} x_{i, j}^{m}
$$

The $\tau$-transformation associated with $P$ is given by

$$
x_{i, j}^{\prime}=\frac{x_{i, j}^{m}}{\sum_{h=1}^{n_{i}} x_{i, j}^{m}} .
$$

The inverse of $\tau$ restricted to $\widetilde{M}$ is given by

$$
x_{i, j}=\frac{x_{i, j}^{\prime 1 / m}}{\sum_{h=1}^{n_{i}} x_{i, h}^{1 / m}}
$$

where the real positive $m$ th roots are to be chosen.

2. The existence of invariants.

2.1. Notation and definitions. As above, we let $\widetilde{M}$ denote the space of real vectors $\left(x_{1,1}, \cdots, x_{1, n_{1}}, \cdots, x_{k, 1}, \cdots, x_{x, n_{k}}\right)$ satisfying $x_{i, j} \geqq$ 0 , and

$$
\sum_{j=1}^{n_{i}} x_{i, j}=1
$$

Let $M$ be set of real vectors

$$
\left(y_{1,1}, \cdots, y_{1, n_{1}^{-1}}, \cdots, y_{k, 1}, \cdots, y_{k, n}\right)
$$

satisfying $y_{i, j} \geqq 0$ and

$$
\sum_{j=1}^{n_{i}-1} y_{i, j} \leqq 1
$$

If $y \in M$ let $\psi(y)$ be the point of $M$ with coordinates $x_{i, j}=y_{i, j}$ for $1 \leqq j \leqq n_{i-1}$ and

$$
x_{i, n_{i}}=1-\sum_{j=1}^{n_{i}-1} y_{i, j}
$$

Clearly $\psi$ is a homeomorphism of $M$ onto $\tilde{M}$.

Let $\varphi$ be a transformation of a set $S$ onto itself. We inductively define $\varphi^{n}(x)$ for $n \geqq 0$ and $x \in S$ by $\varphi^{0}(x)=x$ and $\varphi^{n}(x)=\psi\left(\psi^{n-}(x)\right)$. If $\varphi$ is a one-to-one transformation of $S$ onto itself, we inductively define $\varphi^{n}(x)$ for $n<0$ and $x \in S$ by the rule $\varphi^{n-1}(x)=\varphi^{-1}\left(\varphi^{n}(x)\right)$. Also, 
if $\phi$ is a one-to-one transformation of $S$ onto itself, we have $\varphi^{r+s}(x)=$ $\varphi^{r}\left(\varphi^{s}(x)\right)$ for all $x \in S$ and all pairs of integers $(r, s)$.

Let $\left\{x_{n}\right\}$ be a sequence of points of a topological space $S$. A cluster point of $\left\{x_{n}\right\}$ is a point $p$ of $S$ such that every neighborhood of $p$ contains infinitely many elements of the sequence $\left\{x_{n}\right\}$.

\subsection{Proof of the existence theorem.}

LEMMA 2.1. The transformation $T=\psi^{-1} \tau_{\psi}$ of $M$ into itself has the following properties:

(i) Let $\bar{P}$ be the polynomial defined on $M$ by the formula $\bar{P}(y)=$ $P(\psi(y))$ for $y \in M$. If $y \in M$, either $y=T(y)$ or $\bar{P}(T(y))>\bar{P}(y)$.

(ii) The set of fixed points of $T$ on $M$ is the union of the set of critical points of $\bar{P}$ on $M$ and the sets of critical points of $\bar{P}$ restricted to boundary simplices of $M$.

(iii) The set of fixed points $T$ in $M$ has only finitely many components. Each component of the set of fixed points of $T$ is compact and $\bar{P}$ is constant on each of the components of the set of fixed points of $T$.

(iv) $T$ is a homeomorphism of $M$ onto itself if and only if $\tau$ is a homeomorphism of $M$ onto itself.

(v) If $x \in M$, every cluster point of a sequence $\left\{T^{n}(x)\right\}, n \geqq 0$, is a fixed point of $T$. If $T$ is a homeomorphism, every cluster point of the sequence $\left\{T^{n}(x)\right\}$ is a fixed point of $T$.

Proof. To prove (i), let $y$ be an element of $M$ such that $T(y) \neq$ $y$. Then $\psi^{-1} \tau \psi(y) \neq y$ and $\tau \psi(y) \neq \psi(y)$. Thus $\psi(y)$ is not a fixed point of $\tau$ and it follows that

$$
\bar{P}(T(y))=P\left(\psi \psi^{-1} \tau \psi(y)\right)=P(\tau \psi(y))>P(\psi(y))=\bar{P}(y) .
$$

Statement (ii) may be well known but include a proof for the sake of completeness. Note first that $\psi$ maps the set of fixed points $T$ onto the set of fixed points of $\tau$. Let $x$ be a fixed point of $\tau$ in $\widetilde{M}$ and let $x$ have coordinates $\left(x_{i, j}\right)$. The equation $\tau(x)=x$ implies the equations

$$
x_{i, j}\left(\sum_{1}^{n_{i}} x_{i, k} \frac{\partial P}{\partial x_{i, k}}-\frac{\partial P}{\partial x_{i, j}}\right)=0
$$

for all $i, j$, and since $\tau$ is defined at $x$, these equations imply $\tau(x)=$ $x$. If $x$ is an interior fixed point of $M$, no $x_{i, j}$ is zero so that $\tau(x)=x$ is equivalent to

$$
\frac{\partial P}{\partial x_{i, j}}-\frac{\partial P}{\partial x_{i, n_{i}}}=0
$$


for all $i, j$. But this just the condition that $\psi^{-1}(y)$ be a critical point of $\bar{P}$. Thus the fixed points of $T$ interior to $M$ are just the interior critical points of $\bar{P}$.

Now suppose $y$ is a fixed point of $T$ on the boundary of $M$. Clearly $\psi(y)$ is a fixed point of $\tau$ on the boundary of $\tilde{M}$. If $\psi(y)=z=\left(z_{i, j}\right)$, certain variables $x_{i, i}$ are zero at $z$. Let $\tilde{M}_{y}$ be the part of the boundary of $\tilde{M}$ determined by the equations $x_{i, j}=0$ for all $i, j$ such that $z_{i, j}=0$. If no $z_{i, n_{i}}$ is zero, it follows as before that $y$ is a critical point of $\bar{P}$ restricted to $M_{y}=\psi^{-1}\left(\widetilde{M}_{y}\right)$. Note that $M_{y}$ is a subset of the boundary of $M$. If some $z_{i, n_{i}}$ is zero, the variables $u_{i, j}$ describing $M_{y}$ are subject to the additional constraint $\sum u_{i, j}=1$, where the sum is over the subscripts $i, j$ such that $z_{i, j} \neq 0$. Since the partial derivatives $\partial P / \partial x_{i, j}(z)$ are equal for $i, j$ such that $z_{i, j} \neq 0$, it follows that $y$ is a critical point of $\bar{P}$ for $\bar{P}$ restricted to $M_{y}$. Conversely, if $y$ is a critical point of $P$ restricted to $M_{y}$, it follows that $y$ is a fixed point of $T$.

Let us prove (iii). Let $R_{d}$ be $d$-dimensional real space, with coordinates $x_{i, j}$ as described in the introduction. Let $P$ be a polynomial defined on $R_{d}$. Let $S_{1}$ be the set of points of $R_{d}$ satisfying the equations:

$$
\sum_{j=1}^{n_{i}} x_{i, j}^{2}=1 \text { for all } i, \text { and } \frac{\partial P}{\partial x_{i, j}}=\frac{\partial P}{\partial x_{i, n_{i}}}
$$

for all $i, j$, where the partial derivatives of $P$ are evaluated at $\left(x_{1,1}^{2}, \cdots, x_{1, n_{1}}^{2}, \cdots, x_{k, n_{k}}^{2}\right)$. According to $H$. Whitney [5], a real algebraic variety such as $S_{1}$ has only finitely many components and each component is a union of finitely many components of differentiable manifolds (of various dimensions). Let $Q=P\left(x_{1,1}^{2}, \cdots, x_{k, n_{k}}^{2}\right)$. The partial derivatives of $Q$ with respect to $x_{i, j}$ for $j<n_{i}$ with the restrictions

$$
\sum_{j=1}^{n_{i}} x_{i, j}^{2}=1, i=1, \cdots, k
$$

are all zero on $S_{1}$. Thus $Q$ can have only one value on a component of a differentiable manifold contained in $S_{1}$, and thus can have only finitely many values on $S_{1}$. Since $Q$ is continuous and the components of $S$ are arcwise connected, $Q$ must be constant on each component of $S_{1}$.

Let $\varphi$ be the mapping of $R_{d}$ into itself given by $\varphi\left(x_{1,1}, \cdots, x_{k, n_{k}}\right)=$ $\left(x_{1,1}^{2}, \cdots, x_{k, n_{k}}^{2}\right)$. The set $S=\varphi\left(S_{1}\right)$ is given by the relations:

(i) $x_{i, j} \geqq 0$ for all $i, j$,

(ii) $\sum_{j=1}^{n} x_{i, j}=1$ for $i=1, \cdots, k$, and

(iii) $\partial P / \partial x_{i, j}=\partial P / \partial x_{i, n_{i}}$ (evaluated at $\left(x_{1,1}, \cdots, x_{k, n_{k}}\right)$ ) for all $i, j$. Since $\varphi$ is continuous, $S$ can have only finitely many components. Since $Q(x)=P(\varphi(x))$ for all $x \in R_{d}$, the range of $P$ on $S$ is then range 
of $Q$ on $S_{1}$. Hence $P$ assumes only finitely many values on $S$, and by continuity of $P, P$ is constant on each component of $S$. Since $S$ is just the $\psi$ image of the set of critical points of $\bar{P}$ on $M, S$ is the $\psi$ image of the subset of fixed points of $T$ corresponding to these critical points.

The same argument applies to the sets of critical points of $\bar{P}$ restricted to the boundary sets of $M$ given by certain $x_{i, j}=0$. Since the set $F$ of fixed points of $T$ is the union of the set of critical points of $\bar{P}$ on $M$ and the sets of critical points of $\bar{P}$ restricted to each of finitely many subsets of the boundary of $M, F$ has just finitely many components, and $\bar{P}$ assumes only finitely many values on $F$. By continuity, $\bar{P}$ is constant on each component of $F$. Since $F$ is compact, each of its finitely many components is also compact.

Part (iv) of the lemma follows from the fact that $\psi$ is a homeomorphism of $M$ onto $\tilde{M}$. Since $T=\psi^{-1} \tau \psi, T$ is a homeomorphism of $M$ onto $M$ if $\tau$ is a homeomorphism of $\widetilde{M}$ onto $\tilde{M}$. Since $\tau=\psi T \psi^{-1}$, the converse follows.

The final result, (v), follows directly from the Baum-Eagon inequality (c.f. Section 1 of this paper), and Lemma 2.1 of Bhatia-Szego [3].

In the following, we restrict our attention to those transformations $\tau$ for which $\tau$ is a homeomorphism of $M$ onto itself and $T$ is a homeomorphism of $M$ onto itself.

There is an obvious relation between the functions $f$ defined on $M$ such that $f(T(x))=f(x)$ for all $x$ in $M$ and the functions $g$ defined on $\widetilde{M}$ such that $g(\tau(y))=g(y)$ for all $y \in \tilde{M}$. If $f(T(x))=f(x)$ for all $x \in M$ then $g(y)=f(\psi(y))$ is such that

$$
g(\tau(y))=f\left(\psi \tau \psi^{-1} \cdot \psi(y)\right)=f(T \psi(y))=f(\psi(y))=g(y) .
$$

Conversely, if $g(\tau(y))=g(y)$ it is clear that $f(x)=g\left(\psi^{-1}(x)\right)$ is such that $f(T(x))=f(x)$. Thus we can find all invariant functions of $\tau$ from the invariant functions of $T$.

A spherical neighborhood of a point $x$ of the interior of $M$ is a $d-k$ dimensional ball contained in $M$ with center at $x$. If $x$ is on the boundary of $M$ in $d-k$ dimensional real space, a spherical neighborhood of $x$ in $M$ is the intersection of $M$ and an $d-k$ dimensional ball with center at $x$.

LEMMA 2.2. Let $T$ be a homeomorphism of $M$ onto itself. If $x_{0}$ is a point of $M$ but not a fixed point of $T$, there is a spherical neighborhood $N$ of $x_{0}$ in $M$ such that the sets $T^{r}(N)$ are disjoint for $-\infty<$ $r<\infty$.

Proof. Since $x_{0}$ is not a fixed point of $T, T\left(x_{0}\right) \neq x_{0}$. By Lemma 
1, (i) $\bar{P}\left(T\left(x_{0}\right)\right)-\bar{P}\left(x_{0}\right)=\Delta>0$. Since $\bar{P}$ is continuous on $M$, there is a neighborhood $U$ of $x_{0}$ such that $\bar{P}(x)<\bar{P}\left(x_{0}\right)+\Delta / 3$ for all $x \in U$ and a neighborhood $V$ of $\tau\left(x_{0}\right)$ such that $\bar{P}(y)>\bar{P}\left(T\left(x_{0}\right)\right)-\Delta / 3$ for all $y \epsilon$ $V$. Since $T$ is a continuous transformation, $T^{-1}(V) \cap U$ is a neighborhood of $x_{0}$. Let $N$ be a spherical neighborhood of $x_{0}$ contained in $T^{-1}(V) \cap U$. Since $N \subset U$ and $T(N) \subset V$, for arbitrary $x \in N, y \in T(N)$ we have

$$
\bar{P}(x)<\bar{P}\left(x_{0}\right)+\frac{\Delta}{3} \bar{P}\left(T\left(x_{0}\right)\right)-\frac{\Delta}{3}<\bar{P}(y) .
$$

If $x \in N$ and $z \in T^{m}(N)$ for $m \geqq 1, z=T^{m}(u)$ for some $u \in N$ and $\bar{P}(z) \geqq$ $\bar{P}(T(u))>\bar{P}(x)$ since $T(u) \in T(N)$. Thus $T^{m}(N) \cap N$ is empty for $m \geqq 1$.

Suppose $T^{r}(N) \cap T^{s}(N)$ is not empty for $r \neq s$. We assume $r>s$ and let $y \in T^{r}(N) \cap T^{s}(N)$. Then $T^{-r}(y) \in N$ and $T^{r-s}\left(T^{-r}(y)\right)=T^{-s}(y) \in N$ so that $N$ and $T^{r-s}(N)$ intersect. This contradiction shows that $T^{r}(N) \cap T^{s}(N)$ is empty for $r \neq s$.

If $x, y \in M$, let $|x-y|$ denote the Euclidean distance between $x$ and $y$.

LEMma 2.3. Let $T$ be a homeomorphism of $M$ onto itself. There is a positive number $\varepsilon$ such that if $x$ is a point of $M$ but not a fixed point of $T$, there is at least one element of the sequence $\left\{T^{n}(x)\right\}$ at distance greater than or equal to $\varepsilon$ from the set of fixed points of $T$.

It follows from Baum and Sell [2] that the set $F$ of fixed points of $T$ is an asyptotically stable set. This Lemma is a consequence of Theorem 4.19 of Bhatia-Szego [3].

A fundamental set $S$ for $T$ on $M$ is a subset of $M$ defined as follows: $S$ contains no fixed point of $T$ but if $x$ is not a fixed point of $T, T^{n}(x) \in S$ for a single integer $n$ depending on $S$ and $x$.

Lemma 2.4. If $T$ is a homeomorphism of $M$ onto itself, $T$ has a measurable fundamental set.

Proof. Let $D_{\varepsilon}$ be the set of points of $M$ at distance greater than or equal to $\varepsilon$ from $F$, the set of fixed points of $T$. According to Lemma 2.3, $\varepsilon>0$ may be chosen so that $D_{\varepsilon}$ contains at least one element of every sequence $\left\{T^{n}(x)\right\}$ for $x \notin F$. Since $D_{\varepsilon}$ does not meet $F$, it follows from Lemma 2.2 that about each $x \in D_{\varepsilon}$ there is a spherical neighborhood $N_{x}$ such that the sets $T^{n}\left(N_{x}\right)$ are disjoint (if $x$ is a boundary point of $M$, the set $N_{y}$ is the intersection of a ball with $M$ ). Since $D_{\varepsilon}$ is compact, it is compact relative to $M$ so that there may 
be selected a finite covering $N_{1}, \cdots, N_{r}$ of $D_{\varepsilon}$ from the sets $N_{x}$. Clearly, each sequence $\left\{T^{n}(x)\right\}$ for $x \in M-F$ can meet an $N_{i}$ in at most one point.

Let

$$
\begin{gathered}
L_{1}=N_{1}, L_{2}=N_{2}-\bigcup_{-\infty}^{+\infty} T^{n}\left(N_{1}\right), \cdots \\
L_{r}=N_{r}-\bigcup_{-\infty}^{+\infty} T^{n}\left(N_{1}\right)-\bigcup_{-\infty}^{+\infty} T^{n}\left(N_{2}\right)-\cdots-\bigcup_{-\infty}^{+\infty} T^{n}\left(N_{r-1}\right) .
\end{gathered}
$$

Clearly $\bigcup_{1}^{r} L_{i}$ is a fundamental set for $T$ in $M$. Since $T$ is continuous and each $N_{i}$ is measurable, $\bigcup_{-\infty}^{+\infty} T^{n}\left(N_{i}\right)$ is measurable. Hence each $L_{i}$ is measurable and $U_{1}^{r} L_{i}$ is measurable.

Let $\widetilde{F}$ be the set of fixed points of $\tau$ in $M$.

THeOREM 1. If $T$ is a homeomorphism of $M$ onto itself, and $F$ is the set of fixed points of $T$, there exist $d-k T$-invariant functions of $T$ which are continuous and independent on an open dense subset of $M-F$. Thus there are $d-k \tau$ invariant functions continuous and independent on an open dense subset of $\widetilde{M}-\widetilde{F}$.

Proof. Let $S$ be a fundamental set for $T$ on $M$, as constructed in the proof of Lemma 3.4. Let $S^{*}$ be the boundary of $S$ and let $B=\bigcup_{-\infty}^{+\infty} T^{n}\left(S^{*}\right)$. Then $M-F-B$ is dense in $M-F$. For $x \in M-F$ let $\varphi(x)$ be the element of $\left\{T^{n}(x)\right\}$ in $S$. We will show that $\varphi$ is continuous on $M-F-B$.

If $x \in M-F-B, \varphi(x)$ is the unique intersection of $\left\{T^{n}(x)\right\}$ with $S$. Hence there is an integer $m$ such that $T^{m}(x) \in S$. Since $x \notin B, T^{m}(x)$ is an interior point of $S$. Let $U$ be a neighborhood of $T^{m}(x)$ in $S$. Since $T^{m}$ is continuous, $V=\left(T^{m}\right)^{-1}(U)=T^{-m}(U)$ is a neighborhood of $x$. If $y \in V, T^{m}(y) \in S$ so that $\varphi(y)=T^{m}(y)$ for all $y \in V$. Hence $\varphi$ is continuous in a neighborhood of $x \in M-F-B$, and $M-F-B$ is open. Clearly, $\varphi=T^{m}$ for some $m$ in a neighborhood of $x \in M-F-B$. If we set $\varphi(x)=\left(f_{11}(x), \cdots, f_{1, n_{1}-1}(x), \cdots, f_{k, n_{k-1}}(x)\right)$ so that the $f_{i, j}(x)$ are the components of $\varphi(x)$, it follows that the $f_{i, j}(x)$ are continuous and independent on $M-F-B$, since $\varphi(x)$ is a local homeomorphism on $M-F-B$. Since $\varphi(T(x))=\varphi(x), f_{i, j}(T(x))=f_{i, j}(x)$ so the $f_{i, j}$ are $T$-invariant.

3. The construction of invariant functions. In order to construct invariant functions, we will use more information about sequences $\left\{T^{n}(x)\right\}$ for $x$ not a fixed point of $T$ in $M$. As above, we assume that $T$ is a homeomorphism of $M$ onto itself. For $x \in M$, let 
$L_{x}$ be the set of cluster points of $\left\{T^{n}(x) \mid n>0\right\}$ and let $l_{x}$ be the set of cluster points of $\left\{T^{n}(x) \mid n<0\right\}$. Note that $L_{x}$ and $l_{x}$ are respectively the $\omega$ and $\alpha$ limit sets of $x$.

LEMMA 3.1. The set of cluster points of $\left\{T^{n}(x)\right\}$ is the union of $l_{x}$ and $L_{x}$. The value of $\bar{P}$ is constant on each of $l_{x}$ and $L_{x}$. If $\bar{P}\left(L_{x}\right)$ denotes the value of $\bar{P}$ on $L_{x}$ and $\bar{P}\left(l_{x}\right)$ denotes the value of $\bar{P}$ on $l_{x}$ we have $\bar{P}\left(L_{x}\right)>\bar{P}\left(l_{x}\right)$ whenever $x$ is not a fixed point of $T$ in $M$.

The proof of Lemma 3.1 is straightforward.

Lemma 3.2. Let $x_{0}$ be an element of $M$. Either there is a neighborhood $N$ of $x_{0}$ such that $\left.\bar{P}\left(L_{x}\right)\right)=\bar{P}\left(L_{x_{0}}\right)$ for all $x \in N$ or in every neighborhood of $x_{0}$ there is an $x$ such that $\bar{P}\left(L_{x}\right)>\bar{P}\left(L_{x_{0}}\right)$.

Proof. Suppose there is a neighborhood $N_{1}$ of $x_{0}$ in $M$ such that $\bar{P}\left(L_{x_{0}}\right) \geqq \bar{P}\left(L_{x}\right)$ for all $x \in N_{1}$. Let $\eta$ be a positive number. Let $S^{\eta}$ be the set given by $S_{\eta}=\left\{x \mid \bar{P}\left(L_{x}\right)>\bar{P}\left(L_{x_{0}}\right)-\eta\right\}$. We will show that each $S_{\eta}$ is open. If $x$ is an element of $S_{\eta}$, there is an $m$ such that $\bar{P}\left(T^{m}(x)\right)>\bar{P}\left(L_{x_{0}}\right)-\eta$. Since $T^{m}$ is continuous, there is a neighborhood $N_{x}$ of $x$ such that $\bar{P}\left(T^{m}(y)\right)>\bar{P}\left(L_{x_{0}}\right)-\eta$ for all $y$ in $N_{x}$. But $\bar{P}\left(L_{y}\right) \geqq \bar{P}\left(T^{m}(y)\right)$ for all $y \in M$ so that $\bar{P}\left(L_{y}\right) \in S_{\eta}$ for all $y$ in $N_{x}$. Hence $S_{\eta}$ is open. Let $N(\eta)=S_{\eta} \cap N_{x_{0}}$. Since $x_{0}$ is an element of $S_{\eta}$ for all positive $\eta, N(\eta)$ is not empty for $\eta>0$. Since $N(\eta)$ is contained in $N_{x_{0}}$ and $S_{\eta}, \bar{P}\left(L_{x_{0}}\right) \geqq \bar{P}\left(L_{x}\right) \geqq \bar{P}\left(L_{x_{0}}\right)-\eta$ for all $x$ in $N(\eta)$. Since the points of $L_{x}$ are in $F$, the set of fixed points of $T, \bar{P}\left(L_{x}\right)$ can assume only finitely many values. Hence for $\eta$ sufficiently small

$$
\bar{P}\left(L_{x_{0}}\right) \geqq \bar{P}\left(L_{x}\right) \geqq \bar{P}\left(L_{x_{0}}\right)-\eta
$$

implies that $\bar{P}\left(L_{x}\right)=\bar{P}\left(L_{x_{0}}\right)$, and so for some $\eta, x \in N(\eta)$ implies that $P\left(L_{x}\right)=\bar{P}\left(L_{x_{0}}\right)$.

Lemma 3.3. Let $x_{0}$ be an element of $M$. Either there is a neighborhood $N_{x_{0}}$ of $x_{0}$ in $M$ such that $\bar{P}\left(L_{x_{0}}\right)=\bar{P}\left(L_{x}\right)$ for all $x$ in $N_{x_{0}}$ or every neighborhood $N$ of $x_{0}$ contains an open subset $\Phi_{N}$ such that $P\left(L_{y}\right)=P\left(L_{z}\right)$ for all $y$ and $z$ in $\Phi_{N}$.

Proof. Suppose $x_{0}$ is an element of $M$ and there is no neighborhood $U$ of $x_{0}$ in $M$ such that $\bar{P}\left(L_{x}\right)=\bar{P}\left(L_{x_{0}}\right)$ for all $x$ in $U$. Let $N$ be a neighborhood of $x_{0}$. According to Lemma 3.2, there is an element $x$ of $N$ such that $\bar{P}\left(L_{x}\right)>\bar{P}\left(L_{x_{0}}\right)$. Let $K$ be the least upper bound of $\bar{P}\left(L_{x}\right)$ for $x$ in $N$. Since the range of $\bar{P}\left(L_{x}\right)$ is finite, there is a point $y$ of $N$ such that $\bar{P}\left(L_{y}\right)=K$. Thus $\bar{P}\left(L_{y}\right) \geqq P\left(L_{x}\right)$ for all $x$ in 
$N$, and $N$ is a neighborhood of $y$. By Lemma 3.2, there is a neighborhood $U$ of $y$ such that $\bar{P}\left(L_{y}\right)=\bar{P}\left(L_{x}\right)$ for all $x$ in $U$. Let $\Phi_{N}=$ $N \cap U$.

Using the fact that if $T$ is a homeomorphism of $M$ onto itself, $T^{-1}$ is defined and either $x=T^{-1}(x)$ or $\bar{P}\left(T^{-1}(x)\right)<\bar{P}(x)$, we can modify the above arguments to prove a similar lemma about the function $\bar{P}\left(l_{x}\right)$.

Lemma 3.4. Let $x_{0}$ be an element of $M$. Either there is a neighborhood $N_{x_{0}}$ of $x_{0}$ in $M$ such that $\bar{P}\left(l_{x_{0}}\right)=\bar{P}\left(l_{x}\right)$ for all $x$ in $N_{x_{0}}$, or every neighborhood $N$ of $x_{0}$ contains an open subset $\varphi_{N}$ such that $\bar{P}\left(l_{y}\right)=\bar{P}\left(l_{z}\right)$ for all $y$ and $z$ in $\varphi_{N}$.

THEOREM 2. There is an open dense subset $G$ of $M-F$ such that for any function $f$ continuous on $M$, the series

$$
\sum_{-\infty}^{+\infty} f\left(T^{n}(x)\right)\left[\bar{P}\left(T^{n}(x)\right)-\bar{P}\left(T^{n-1}(x)\right)\right]
$$

represents a $T$ invariant function continuous on $G$.

Proof. Let $G_{1}$ be the set of all elements $x$ of $M$ such that $\bar{P}\left(L_{x}\right)$ is constant in a neighborhood of $x$. Let $G_{2}$ be the set of all elements $x$ of $M$ such that $\bar{P}\left(l_{x}\right)$ is constant in a neighborhood of $x$. Clearly, $G_{1}$ and $G_{2}$ are open relative to $M$ and by Lemmas 3.3 and 3.4, each of $G_{1}$ and $G_{2}$ is dense in $M$. Hence $G=(M-F) \cap G_{1} \cap G_{2}$ is an open dense subset of $M-F$.

For each $x$ in $M$ let $S(x)$ denote the series

$$
S(x)=\sum_{-\infty}^{+\infty} \bar{P}\left(T^{n}(x)\right)-\bar{P}\left(T^{n-1}(x)\right) \text {. }
$$

Now $S(x)$ converges at each $x$ to $\bar{P}\left(L_{x}\right)-\bar{P}\left(l_{x}\right)$.

Let $y$ be an element of $G$. There is a neighborhood $U$ of $y$ such that $S(x)$ represents the constant function in $U$. Since $y \notin F$ and $F$ is compact, there is a neighborhood $V$ of $y$ containing no fixed points of $T$. Let $W$ be a neighborhood of $y$ such that $\bar{W} \subset U \cap V$. Now $S(x)$ is a series of positive terms converging to a continuous function on $\bar{W}$, and so by E. C. Titchmarsh [4], art. 1.31, $S(x)$ converges uniformly on $\bar{W}$. Let $f(x)$ be any function continuous on $M$. The series

$$
F(x)=\sum_{-\infty}^{+\infty} f\left(T^{n}(x)\right)\left[\bar{P}\left(T^{n}(x)\right)-\bar{P}\left(T^{n-1}(x)\right]\right.
$$

converges uniformly on $\bar{W}$ since $f$ is bounded on $M$. Since $f, \bar{P}$ and 
$T$ are continuous, $F(x)$ is continuous on $\bar{W}$ and hence at $y$. Clearly $F(T(x))=F(x)$, so the function $F$ is a continuous $T$ invariant function on $G$.

We initiate the study of differentiable $T$ invariant functions by defining certain series of continuous functions on $G$, the set defined in the proof of Lemma 3.4. Recall that a point $x_{0}$ of $M$ is a point of $G$ if and only if $x_{0}$ is not a fixed point of $T$ and there is a neighborhood $N$ of $x_{0}$ such that the functions $\bar{P}\left(L_{x}\right)$ and $\bar{P}\left(l_{x}\right)$ are constant on $N$.

LEMMA 3.5. If a function $h(x)$ is defined on all of $M-F$ by the formula

$$
h(x)=\frac{2\left(\bar{P}\left(L_{x}\right)-\bar{P}(x)\right)+\frac{1}{2}\left(\bar{P}(x)-\bar{P}\left(l_{x}\right)\right)}{\bar{P}\left(L_{x}\right)-\bar{P}\left(l_{x}\right)},
$$

then $h(x)$ has the following properties:

(i) $h(x)$ is defined and nonnegative on $M-F$,

(ii) $h(x)$ is continuous at every point of $G$, and

(iii) if $x_{0}$ is a point of $G$, there is a neighborhood $V$ of $x_{0}$ such that $\bar{V}$ is contained in $G$, and an integer $m>0$ such that

$$
h\left(T^{-n}(x)\right)>\frac{7}{4}
$$

and

$$
0<h\left(T^{n}(x)\right)<\frac{3}{4}
$$

for all $n>m$ and $x \in \bar{V}$.

Proof. If $x_{0}$ is an element of $M-F, x_{0}$ is not a fixed point of $T$ and hence $\bar{P}\left(L_{x_{0}}\right)-\bar{P}\left(l_{x_{0}}\right)>0$. Hence $h(x)$ is defined on $M-F$. Since $\bar{P}\left(\left(L_{x}\right)>\bar{P}(x)\right.$ and $\bar{P}(x)>\bar{P}\left(l_{x}\right)$ for $x$ in $M-F, h(x)$ is positive on $M-F$. To prove (ii), let $x_{0}$ be a point of $G$. By the definition of $G$, there is a neighborhood $N_{1}$ of $x_{0}$ such that $\bar{P}\left(L_{x}\right)$ and $\bar{P}\left(l_{x}\right)$ are constant on $N_{1}$. By the definition of $G, x_{0}$ is not a fixed point of $T$ so that $P\left(L_{x_{0}}\right)-\bar{P}\left(l_{x_{0}}\right)>0$. Hence $\bar{P}\left(L_{x}\right)-\bar{P}\left(l_{x}\right)$ is a nonzero constant on $N_{1}$. Since $\bar{P}(x), \bar{P}\left(L_{x}\right)$ and $\bar{P}\left(l_{x}\right)$ are continuous in $N_{1}, h(x)$ is continuous in $N_{1}$ and hence at $x_{0}$.

To prove (iii), let $x_{0}$ be a point of $G$ and let $N_{1}$ be a neighborhood of $x_{0}$ such that $\bar{P}\left(L_{x}\right)$ and $\bar{P}\left(l_{x}\right)$ are constant on $N_{1}$. Then $G \supset$ $N_{1}$. Let $V$ be neighborhood of $x_{0}$ such that $\bar{V} \subset N_{1} \subset G$. As in the 
proof of (ii), $h(x)$ is continuous on $N_{1}$ and hence on $\bar{V}$. Since $T$ is a homeomorphism of $M$ onto itself, $T^{n}$ is a continuous transformation of $M$ onto itself for arbitrary integral $n$. Hence $h\left(T^{n}(x)\right)$ is continuous on $\bar{V}$ for arbitrary integral $n$. Let $n$ be an integer. Since $\bar{P}\left(L_{T(x)}\right)=$ $\bar{P}\left(L_{x}\right)$ and $\bar{P}\left(l_{T(x)}\right)=\bar{P}\left(l_{x}\right)$ the difference between $h\left(T^{n+1}(x)\right)$ and $h\left(T^{n}(x)\right)$ is given by the formula

$$
h\left(T^{n+1}(x)\right)-h\left(T^{n}(x)\right)=-\frac{\frac{3}{2}\left[\bar{P}\left(T^{n+1}(x)\right)-\bar{P}\left(T^{n}(x)\right)\right]}{\bar{P}\left(L_{x}\right)-\bar{P}\left(l_{x}\right)} .
$$

No point of $N_{1}$ is a fixed point of $T_{n}$ since

$$
\bar{P}\left(L_{T^{n}(x)}\right)-\bar{P}\left(l_{T^{n}(x)}\right)=\bar{P}\left(L_{x}\right)-\bar{P}\left(l_{x}\right)
$$

and

$$
\bar{P}\left(L_{x}\right)-\bar{P}\left(l_{x}\right)=\bar{P}\left(L_{x_{0}}\right)-\bar{P}\left(l_{x_{0}}\right)>0
$$

Hence

$$
h\left(T^{n+1}(x)\right)<h\left(T^{n}(x)\right)
$$

for all $x$ in $\bar{V}$ and all integers $n$. Hence $h\left(T^{n}(x)\right)$ is a monotone decreasing function of $n$ for each $x$ in $\bar{V}$. Since $\lim _{n \rightarrow \infty} h\left(T^{n}(x)\right)=1 / 2$ and $\lim _{n \rightarrow-\infty} h\left(T^{n}(x)\right)=2$ for all $x$ in $\bar{V}$, it follows from the compactness of $\bar{V}$ that there is an integer $m$ such that

$$
2 \geqq h\left(T^{-n}(x)\right)>\frac{7}{4}
$$

and

$$
\frac{3}{4}>h\left(T^{n}(x)\right) \geqq \frac{1}{2}
$$

for all integers $n>m$ and all elements $x$ of $\bar{V}$.

LEMMA 3.6. Let $h(x)$ be the function defined in Lemma 3.5. Let the sequence $p_{n}(x)$ be inductively defined for integral $n$ by the rules:

(i) $p_{0}=1$

(ii) $p_{n+1}(x)=h\left(T^{n}(x)\right) p_{n-1}(x)$ for $n \geqq 1$

(iii) $p_{-n}(x)=p_{-n+1}(x) / h\left(T^{-n}(x)\right)$ for $n \geqq 1$.

If $x_{0}$ is an element of $G$ every $p_{n}(x)$ is continuous at $x_{0}$ and there is $a$ neighborhood $V$ of $x_{0}$, a constant $K$ and an integer $m$ such that

$$
0<p_{n}(x)<K \cdot\left(\frac{3}{4}\right)^{|n|-m}
$$

for all $x$ in $\bar{V}$ and all $n$ such that $|n|>m$. 
The proof of Lemma 3.6 is straightforward and has been omitted.

LEMMA 3.7. If $q_{n, r}(x)$ is defined by the formula

$$
q_{n, r}(x)=\frac{p_{n}(x)^{r}}{\sum_{j=-\infty}^{+\infty}\left[p_{j}(x)\right]^{r}}
$$

then

(i) each $q_{n, r}(x)$ is defined and continuous for $x \in G$,

(ii) if $x_{0}$ is an element of $G$, there is a neighborhood $V$ of $x_{0}$ such that $\bar{V} \subset G$, and an integer $m$ such that

$$
0<q_{n, r}(x)<\left(\left(\frac{3}{4}\right)^{r}\right)^{|n|-m}
$$

for all $n$ such that $|n|>m$ and all positive integers $r$.

(iii) for all $x$ in $G, q_{n, r}(T(x))=q_{n+1, r}(x)$,

(iv) if $f(x)$ is a continuous function on $M$, and $r$ and $s$ are positive integers

$$
\sum_{n=-\infty}^{+\infty} f\left(T^{n}(x)\right) q_{n_{1} r}(x)^{s}
$$

defines a continuous T-invariant function on $G$.

Proof. To prove statement (i), let $x_{0}$ be a point of $G$. According to Lemma 3.6, there is a neighborhood $V$ of $x_{0}$ such that $\bar{V} \subset G$ and

$$
0<p_{n}(x)<K \cdot\left(\frac{3}{4}\right)^{|n|-m}
$$

for $n$ sufficiently large. Hence the series

$$
\sum_{-\infty}^{+\infty} p_{n}(x)^{r}
$$

converges uniformly for all $x$ in $\bar{V}$. Since $p_{n}(x)^{r}$ is continuous in $\bar{V}$, and

$$
\sum_{-\infty}^{+\infty} p_{n}(x)^{r}>p_{0}(x)^{r}=1
$$

every $q_{n, r}(x)$ is defined and continuous in $\bar{V}$. Since $x_{0}$ is an arbitrary point of $G$, statement (i) is proven.

To prove statement (ii), let $x_{0}$ be a point of $G$. According to Lemma 3.6, there is a neighborhood $V$ of $x_{0}$ such that $\bar{V} \subset G$, a constant $K$ and an integer $v$ such that

$$
0<p_{n}(x)<K\left(\frac{3}{4}\right)^{|n|-v} .
$$


Let $m$ be so larger that $K \cdot(3 / 4)^{m-v}<1$. Then we have

$$
0<p_{n}(x)<\left(\frac{3}{4}\right)^{|n|-m},
$$

so that

$$
0<p_{n}(x)^{r}<\left(\left(\frac{3}{4}\right)^{r}\right)^{|n|-m}
$$

Since

$$
\sum_{-\infty}^{+\infty} p_{n}(x)^{r}>p_{0}(\alpha)^{r}=1
$$

we can obtain the inequality of (ii).

Statement (iii) follows directly from the observation that whenever $p_{n}(x)$ is defined, we have

$$
p_{n}(T(x))=\frac{p_{n+1}(x)}{h(x)} .
$$

To prove statement (iv) note that wherever all $q_{n, r}(x)$ are defined we have

$$
f\left(T_{n}(T(x))\right) q_{n, r}(T(x))^{s}=f\left(T^{n+1}(x)\right) q_{n+1, r}(x)^{s},
$$

so that the $T$ invariance of the series of (iv) follows. Since $f(x)$ is continuous on $M$ and $M$ is compact, $|f(x)|$ is bounded on $M$. By part (iii), the series of part (iv) converges uniformly in a closed neighborhood of each point of $G$ for all positive integers $r$. Hence if $r$ and $s$ are arbitrary positive integers,

$$
\sum_{n=-\infty}^{+\infty} f\left(T^{n}(x)\right)\left[q_{n, r}(x)\right]^{s}
$$

represents a continuous $T$-invariant function on $G$.

Let $J$ be the Jacobian of the transformation $T$ and let $|J|$ be the determinant of $J$. If $|J|$ is bounded away from zero on $M$, we can construct $T$ invariant functions which are differentiable on an open dense subset of $M-F$. We can show that the hypothesis that $|J|$ is bounded away from zero on $M$ and $T$ is a homeomorphism are reasonable by an example. Let $P$ be any polynomial with positive coefficients defined on $\widetilde{M}$. Let $R$ be the polynomial given by the formula

$$
R=\sum_{i=1}^{k}\left(\sum_{j=1}^{n_{i}} x_{i, j}\right)
$$


and let $Q_{\varepsilon}=R+\varepsilon P$. For $\varepsilon>0, Q_{\varepsilon}$ has positive coefficients and by the unpublished result of L. E. Baum stated above, the $T$ transformation $T_{\varepsilon}$ associated with $Q_{\varepsilon}$ is a homeomorphism of $M$ onto itself. The $T$ transformation associated with $R=Q_{0}$ is the identity transformation so that the determinant of the Jacobian of $T_{0}$ is 1 . If we let $J_{\varepsilon}$ be the Jacobian of $T_{\varepsilon},\left|J_{\varepsilon}\right|$ is a continuous function of $\varepsilon$, and $\left|J_{\varepsilon}\right| \rightarrow 1$ as $\varepsilon \rightarrow 0$ at each point of $M$. Since $M$ is compact, there is an $\varepsilon$ such that $\left|J_{\varepsilon}\right|>1 / 2$ at every point of $M$.

In the following we will assume that $|J|$ is bounded away from zero on $M$, but we note that local results can be obtained by restricting our attention to elements $x$ of $M$ such that $|J|$ is bounded away from zero in some neighborhood of the sequence $\left\{T^{n}(x)\right\}$.

LEMMA 3.8. If $T$ is a homeomorphism of $M$ onto itself, the Jacobian determinant $|\boldsymbol{J}|$ of $T$ is bounded away from zero on $M$ and $t_{n, u, v}(x)$ denotes the $(u, v)$ component of $T^{n}(x)$, then:

(i) for every $n$ and subscript pair $i, j, \partial / \partial x_{i, j}\left(t_{n, u, v}(x)\right)$ is continuous on $M$;

(ii) there is a constant $B$ such that

$$
\left|\frac{\partial}{\partial x_{i, j}} t_{n, u, v}(x)\right|<B^{|n|}
$$

for all $(i, j)$ and all $x$ in $M$;

(iii) if $C$ is a compact subset of $G$ there is a positive integer $r$ such that the first partial derivatives

$$
\frac{\partial}{\partial x_{i, j}} q_{n, r}(x)
$$

(see Lemma 3.7 for the definition of the functions $q_{n, r}(x)$ ) are continuous in $C$ and there are constants $L_{1}$ and $L_{2}$ such that

$$
\left|\frac{\partial}{\partial x_{i, j}} q_{n, r}(x)\right|<L_{1} L_{2}^{\lfloor n !}
$$

and $0<L_{2}<1$, for all $x$ in $C$.

Proof. Since $T^{n}$ is a rational transformation of $M$, with nonzero denominators, $\partial / \partial x_{i, j}\left(t_{n, u, v}(x)\right)$ is continuous on $M$ for all $n \geqq 0$. Since the Jacobian determinant of $T$ is bounded away from zero on $M$, the same result holds for $\partial / \partial x_{i, j}\left(t_{-n, u, v}(x)\right)$.

To prove (ii), note that

$$
\frac{\partial}{\partial x_{i, j}} t_{n, u, v}(x)=\sum_{r, s} \frac{\partial t_{1, u, v}}{\partial x_{r, s}}\left(T^{n-1}(x)\right) \frac{\partial t_{n-1, u, v}(x)}{\partial x_{i, j}}
$$


for all $n$ and every $x$ in $M$. Since

$$
\frac{\partial t_{1, u, v}}{\partial x_{r, s}}(x)
$$

is bounded on $M$ for all $(r, s)$, it follows inductively that there are bounds $L_{1}$ and $R_{1}$ such that

$$
\left|\frac{\partial}{\partial x_{i, j}} t_{n, u, v}(x)\right|<L_{1} \cdot R_{1}^{n}
$$

for all $n>0$. Since the determinant of

$$
J=\left(\frac{\partial}{\partial x_{i, j}} t_{1, u, v}(x)\right)
$$

is bounded away from zero on $M_{1}$ the elements of the matrix $J^{-1}$ are bounded on $M$. It follows that there are constants $L_{2}$ and $R_{2}$ such that

$$
\left|\frac{\partial}{\partial x_{i, j}} t_{n, u, v}(x)\right|<L_{2} \cdot R_{2}^{n}
$$

for all $n \leqq 0$. Clearly there is a constant $B$ such that $B^{|n|}>L_{4} \cdot R_{1}^{\mid n}$ and $B^{|n|}>L_{2} \cdot R_{2}^{|n|}$, so that

$$
\left|\frac{\partial}{\partial x_{i, j}} t_{n, u, v}(x)\right|<B^{|n|}
$$

for all $n, u, v$ and all $x \in M$.

To prove (iii) we will show first that for a given $x_{0} \in G$ there is a closed neighborhood $V_{x_{0}}$ of $x_{0}$ and an integer $i$ such that

$$
\sum_{-\infty}^{+\infty} \frac{\partial}{\partial x_{i, j}}\left[p_{n}(x)\right]^{r}
$$

converges uniformly in $\bar{V}_{x_{0}}$ for all $r \geqq i$. By Lemma 3.6, there is a neighborhood $V$ of $x_{0}$ such that $G \supset \bar{V}$, a bound $K$ and an integer $m$ such that

$$
0<p_{n}(x)<K \cdot\left(\frac{3}{4}\right)^{n-m}
$$

for all $x \in \bar{V}$.

For $n>0$ we will inductively find a bound $S$ such that

$$
\left|\frac{\partial}{\partial x_{i, j}} p_{n}(x)\right|<S^{n+1} \text {. }
$$

We have 


$$
\begin{aligned}
\frac{\partial}{\partial x_{i, j}} p_{n}(x) & =\frac{\partial}{\partial x_{i, j}} h\left(T^{n}(x)\right) p_{n-1}(x) \\
& =h\left(T^{n}(x)\right) \frac{\partial}{\partial x_{i, j}} p_{n-1}(x)+p_{n-1}(x) \sum_{u, v} \frac{\partial}{\partial x_{u, v}} \cdot \frac{\partial t_{n, u, v}}{\partial x_{i, j}}
\end{aligned}
$$

Now $0<h\left(T^{n}(x)\right)<2$ for $x \in \bar{V}$, and there is a bound $B_{1}$ such that $\left|p_{n-1}(x)\right|<B_{1}$ for $x \in \bar{V}$. For every subset of $G$,

$$
\frac{\partial h}{\partial x_{u, v}}=-\frac{3}{2} \frac{\frac{\partial}{\partial x_{u, v}}(\bar{P})}{\bar{P}\left(L_{x}\right)-\bar{P}\left(l_{x}\right)}
$$

is bounded on $G$ since $\bar{P}$ is a polynomial and $\bar{P}\left(L_{x}\right)-\bar{P}\left(l_{x}\right)$ ranges over a finite set not including zero for all $x \in G$. Since $G$ is closed under the transformation $T$, there is a constant $B_{2}$ such that $\left|\partial h / \partial x_{u, v}\right|<$ $B_{2}$ at $T^{n}(x)$ for every element $x$ of $\bar{V}$. Thus

$$
\left|\frac{\partial}{\partial x_{i, j}} p_{n}(x)\right|<2\left|\frac{\partial}{\partial x_{i, j}} p_{n-1}(x)\right|+d B_{1} B_{2} B^{n} .
$$

If $K_{1}$ is maximum of $2, d B_{1} B_{2}$ and $B$ we have

$$
\left|\frac{\partial}{\partial x_{i, j}} p_{n}(x)\right|<K_{1}\left(\left|\frac{\partial}{\partial x_{i, j}} p_{n-1}(x)\right|+K_{1}^{n}\right) \text {. }
$$

Since $p_{0}(x)=1$, we have

$$
\begin{aligned}
& \left|\frac{\partial}{\partial x_{i, j}} p_{1}(x)\right|<K_{1}^{2} \\
& \left|\frac{\partial}{\partial x_{i, j}} p_{2}(x)\right|<2 K_{1}^{3}
\end{aligned}
$$

and

$$
\left|\frac{\partial}{\partial x_{i, j}} p_{n}(x)\right|<n K_{1}^{n+1}<S^{n+1}
$$

for some bound $S$ and all $x$ in $\bar{V}$.

Since $h\left(T^{-n}(x)\right)>1 / 2$ for all $x \in \bar{V}$, a similar argument yields a constant $S_{1}$ such that

$$
\left|\frac{\partial p_{n}}{\partial x_{i, j}}(x)\right|<S_{1}^{|n|+1}
$$

for negative $n$ all $x \in \bar{V}$. Hence there is a single constant $S$ so that

$$
\left|\frac{\partial P_{n}(x)}{\partial x_{i, j}}\right|<S^{|n|+1}
$$


for all $x \in \bar{V}$. Now $\bar{V}$ was selected so that

$$
0<p_{n}(x)<K\left(\frac{3}{4}\right)^{|n|-m}
$$

for $n$ such that $|n|>m$. For $p$ sufficiently large,

$$
0<p_{n}(x)<\left(\frac{3}{4}\right)^{|n|-p}
$$

for all $n$ such that $|n|>p>m$, and so

$$
0<p_{n}(x)^{r-1}<\left(\left(\frac{3}{4}\right)^{r-1}\right)^{|n|-p}
$$

and

$$
\begin{aligned}
\left|\frac{\partial}{\partial x_{i, j}} p_{n}(x)^{r}\right| & =\left|r p_{n}(x)^{r-1}\right|\left|\frac{\partial}{\partial x_{i, j}} p_{n}(x)\right| \\
& \leqq r s\left(S\left(\frac{3}{4}\right)^{r-1}\right)^{p}\left(\left(\frac{3}{4}\right)^{r-1} S\right)^{|n|-p} .
\end{aligned}
$$

Now $r$ can be chosen so large that $S(3 / 4)^{r-1}<1$. Thus there are constants $C$ and $D$ so that $0<D<1 \tau$ and

$$
\left|\frac{\partial}{\partial x_{i, j}} p_{n}(x)^{r}\right| \leqq C D^{|n|-p}
$$

for all $x$ in $\bar{V}$. Hence the series

$$
\sum_{-\infty}^{+\infty} p_{n}(x)^{r}
$$

has continuous first parial derivatives for $x \in \bar{V}$. Since

$$
\sum_{-\infty}^{+\infty} p_{n}(x)^{r}>p_{0}(x)^{r}=1,
$$

we have that $q_{0, r}(x)$ has continuous first partial derivatives for all $x$ in $\bar{V}$. But

$$
q_{n, r}(x)=p_{n}(x)^{r} \cdot q_{0, r}(x)
$$

so that $q_{n, r}(x)$ has continuous first partial derivatives for all $x$ in $\bar{V}$. Since $\bar{V}$ is compact, there is a bound $U$ on the partial derivatives of $q_{0, r}(x)$ in $\bar{V}$. Thus

$$
\frac{\partial}{\partial x_{i, j}} q_{n, r}(x)=q_{0, r}(x) \frac{\partial}{\partial x_{i, j}} p_{n}(x)^{r}+p_{n}(x)^{r} \frac{\partial}{\partial x_{i, j}} q_{0, r}(x)
$$

and 


$$
\begin{aligned}
\left|\frac{\partial}{\partial x_{i, j}} q_{n, r}(x)\right| & \leqq W C D^{|n|-p}+\left(\frac{3}{4}\right)^{r(|n|-p)} \cdot U \\
& \leqq R_{1} \cdot R_{2}^{|n|}
\end{aligned}
$$

with $0<R_{2}<1$.

Since $C$ is compact, it can be covered with a finite set of neighborhoods as $\bar{V}$, so part (iii) of the lemma follows immediately.

THEOREM 3. If $C$ is a compact subset of $G$, there are integers $r$ and $s$ such that for every function $f(x)$, defined and with continuous first partial derivatives on $M$, the function

$$
F(x)=\sum_{-\infty}^{+\infty} f\left(T^{n}(x)\right)\left[q_{n, r}(x)\right]^{s}
$$

is continuous and has continuous first partial derivatives for all $x$ in $\bigcup_{-\infty}^{+\infty} T^{n}(C)$ and

$$
F(x)=F(T(x))
$$

wherever $F(x)$ is defined.

Proof. Clearly $F(x)=F(T(x))$ wherever $F(x)$ is defined. Also, if all first prtial derivatives $\partial / \partial_{x i, j} F(x)$ are defined and continuous at $x=x_{0}$, it follows by elementary methods from the fact that $|J|$ is bounded away from zero on $M$ and $J$ is continuous on $M$ that $\partial / \partial x_{i, j} F(x)$ is defined and continuous at $T^{n}\left(x_{0}\right)$ for all $n$. Hence we need only show that $F(x)$ has continuous first partial derivatives for all elements $x$ of $C$. We choose to show that the series of partial derivatives

$$
\sum_{-\infty}^{+\infty} \frac{\partial}{\partial x_{i, j}} f\left(T^{n}(x)\right)\left[q_{n, r}(x)\right]^{s}
$$

converges uniformly in $C$.

Note that

$$
\begin{aligned}
\frac{\partial}{\partial x_{i, j}} f\left(T^{n}(x)\right)\left[q_{n, r}(x)\right]^{s}= & \left.q_{n, r}(x)^{s} \sum_{u, v} \frac{\partial f}{\partial x_{u, v}}\right|_{T^{n}(x)} \frac{\partial t_{n, u, v}}{\partial x_{i, j}} \\
& +s\left[q_{n, r}(x)\right]^{s-1} \frac{\partial q_{n, r}(x)}{\partial x_{i, j}} f\left(T^{n}(x)\right) .
\end{aligned}
$$

Since $f$ and its first partial derivatives are bounded on $M$ it follows directly from Lemma 3.8 that $s$ may be chosen so large that the series

$$
\sum_{-\infty}^{+\infty} \frac{\partial}{\partial x_{i, j}} f\left(T^{n}(x)\right)\left[q_{n, r}(x)\right]^{s}
$$


is majorized by a geometric series, and the choice of $s$ is independent of $f$.

The analysis of this section can be extended to obtain a local analogue of Theorem 1, i.e., a set of $d-k$ functions can be found which are continuous on $G$, have nonzero Jacobian at a point $x_{0}$ of $G$ and are invariant with respect to $T$.

The author thanks L. E. Baum, D. Birkes, and D. S. Passman for their many stimulating conversations during the conduct of this study.

\section{REFERENCES}

1. L. E. Baum and J. A. Eagon, An inequality with applications to statistical estimation for probabilistic functions of Markov processes and to a model for ecology, Bull. Amer. Math. Soc., 73 (1967), 360-363.

2. L. E. Baum and G. R. Sell, Growth transformations for functions on manifolds, Pacific J. Math., 27, (1968), 211-227.

3. N. P. Bhatia and G. P. Szego, Stability Theory of Dynamical Systems, Springer.

4. E. .C. Titchmarch, The Theory of Functions, Second Edition, Oxford, 1939.

5. H. Whitney, Elementary structure of real algebraic varieties, Annals of Math., 66, (1957), 545-556.

Received February 18, 1971 and in revised form July 20, 1972.

The City College 



\section{PACIFIC JOURNAL OF MATHEMATICS}

\section{EDITORS}

\author{
H. SAMELSON \\ Stanford University \\ Stanford, California 94305 \\ C. R. HOBBY \\ University of Washington \\ Seattle, Washington 98105
}

\section{J. DugundjI}

Department of Mathematics University of Southern California Los Angeles, California 90007

\section{RICHARD ARENS}

University of California

Los Angeles, California 90024

\section{ASSOCIATE EDITORS}
E. F. BECKENBACH
B. H. NeumanN
F. WOLF
K. YosHIDA

\section{SUPPORTING INSTITUTIONS}

\author{
UNIVERSITY OF BRITISH COLUMBIA \\ CALIFORNIA INSTITUTE OF TECHNOLOGY \\ UNIVERSITY OF CALIFORNIA \\ MONTANA STATE UNIVERSITY \\ UNIVERSITY OF NEVADA \\ NEW MEXICO STATE UNIVERSITY \\ OREGON STATE UNIVERSITY \\ UNIVERSITY OF OREGON \\ OSAKA UNIVERSITY
}

\author{
UNIVERSITY OF SOUTHERN CALIFORNIA \\ STANFORD UNIVERSITY \\ UNIVERSITY OF TOKYO \\ UNIVERSITY OF UTAH \\ WASHINGTON STATE UNIVERSITY \\ UNIVERSITY OF WASHINGTON \\ ${ }^{*} \stackrel{*}{*}{ }^{*}$
AMERICAN MATHEMATICAL SOCIETY
NAVAL WEAPONS CENTER
}

The Supporting Institutions listed above contribute to the cost of publication of this Journal, but they are not owners or publishers and have no responsibility for its content or policies.

Mathematical papers intended for publication in the Pacific Journal of Mathematics should be in typed form or offset-reproduced, (not dittoed), double spaced with large margins. Underline Greek letters in red, German in green, and script in blue. The first paragraph or two must be capable of being used separately as a synopsis of the entire paper. The editorial "we" must not be used in the synopsis, and items of the bibliography should not be cited there unless absolutely necessary, in which case they must be identified by author and Journal, rather than by item number. Manuscripts, in duplicate if possible, may be sent to any one of the four editors. Please classify according to the scheme of Math. Rev. Index to Vol. 39. All other communications to the editors should be addressed to the managing editor, Richard Arens, University of California, Los Angeles, California, 90024.

50 reprints are provided free for each article; additional copies may be obtained at cost in multiples of 50 .

The Pacific Journal of Mathematics is published monthly. Effective with Volume 16 the price per volume (3 numbers) is $\$ 8.00$; single issues, $\$ 3.00$. Special price for current issues to individual faculty members of supporting institutions and to individual members of the American Mathematical Society: $\$ 4.00$ per volume; single issues $\$ 1.50$. Back numbers are available.

Subscriptions, orders for back numbers, and changes of address should be sent to Pacific Journal of Mathematics, 103 Highland Boulevard, Berke'ey, California, 94708.

PUBLISHED BY PACIFIC JOURNAL OF MATHEMATICS, A NON-PROFIT CORPORATION

Printed at Kokusai Bunken Insatsusha (International Academic Printing Co., Ltd.), 270, 3-chome Totsuka-cho, Shinjuku-ku, Tokyo 160, Japan. 


\section{Pacific Journal of Mathematics}

\section{Vol. 43, No. $3 \quad$ May, 1972}

Max K. Agoston, An obstruction to finding a fixed point free map on a manifold.... 543

Nadim A. Assad and William A. Kirk, Fixed point theorems for set-valued mappings

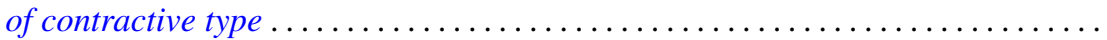

John Winston Bunce, Characterizations of amenable and strongly amenable

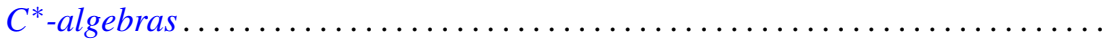

Erik Maurice Ellentuck and Alfred Berry Manaster, The decidability of a class of

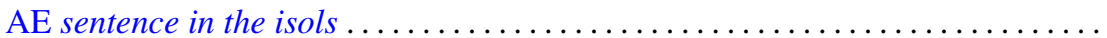

U. Haussmann, The inversion theorem and Plancherel's theorem in a Banach

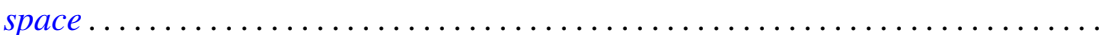

Peter Lawrence Falb and U. Haussmann, Bochner's theorem in infinite dimensions.

Peter Fletcher and William Lindgren, Quasi-uniformities with a transitive base ..... Dennis Garbanati and Robert Charles Thompson, Classes of unimodular abelian

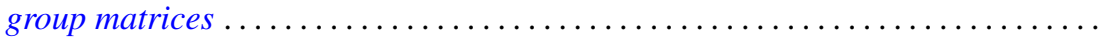

Kenneth Hardy and R. Grant Woods, On c-realcompact spaces and locally bounded normal functions

Manfred Knebusch, Alex I. Rosenberg and Roger P. Ware, Grothendieck and Witt rings of hermitian forms over Dedekind rings .......................

George M. Lewis, Cut loci of points at infinity.

Jerome Irving Malitz and William Nelson Reinhardt, A complete countable $L_{\omega_{1}}^{Q}$ theory with maximal models of many cardinalities . . . . . . . . . . . . . . . . .

Wilfred Dennis Pepe and William P. Ziemer, Slices, multiplicity, and Lebesgue

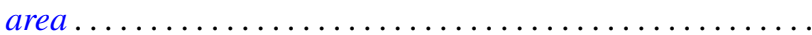

Keith Pierce, Amalgamating abelian ordered groups . .

Stephen James Pride, Residual properties of free groups . . . . . . . . . . . . . 725

Roy Martin Rakestraw, The convex cone of n-monotone functions .

T. Schwartzbauer, Entropy and approximation of measure preserving transformations .

Peter F. Stebe, Invariant functions of an iterative process for maximization of a polynomial...

Kondagunta Sundaresan and Wojbor Woyczynski, L-orthogonally scattered

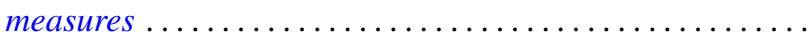

Kyle David Wallace, $C_{\lambda}$-groups and $\lambda$-basic subgroups $\ldots \ldots \ldots$

Barnet Mordecai Weinstock, Approximation by holomorphic functions on certain

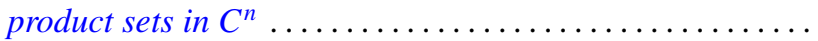

Donald Steven Passman, Corrections to: "Isomorphic groups and group rings”.

Don David Porter, Correction to: "Symplectic bordism, Stiefel-Whitney numbers, and a Novikov resolution"

John Ben Butler, Jr., Correction to: “Almost smooth perturbations of self-adjoint operators".

Constantine G. Lascarides, Correction to: "A study of certain sequence spaces of Maddox and a generalization of a theorem of Iyer" ...... ...

George A. Elliott, Correction to: "An extension of some results of takesaki in the reduction theory of von neumann algebras" ......................... 